\title{
Prognosis of partial epilepsy
}

\author{
G PORRO, M MATRICARDI, V GUIDETTI, AND P BENEDETTI \\ Instituto di Neuropsichiatria, Rome, Italy
}

SUMmaRY The prognosis of partial epilepsy in childhood (excluding cases of benign partial epilepsy) was studied; the average follow up period was 7.4 years. Improvement rate of seizure status was $82 \cdot 3 \%$. We studied favourable prognostic factors and found that those most often associated with seizure improvement were familial convulsions and idiopathic forms, no generalised seizures before partial onset, low frequency of seizures after 12 months of treatment, short duration of epilepsy, and no background activity abnormalities on electroencephalography. We also observed such factors as mental retardation, neurological abnormalities, and behaviour and cognitive disorders. Factors that determined the prognosis for social adjustment were similar to those for seizure improvement. We discuss the favourable prognosis of partial seizures in childhood and the predictive factors.

Partial epileptic seizures in childhood are quite common representing, according to various authors, from $40 \%{ }^{2}$ to $70 \%^{34}$ of the whole range of epileptic forms found among children. The literature on partial epileptic seizures has focused upon the electroclinical characteristics of the single forms, particularly those with complex symptomatology $y^{5-8}$ and on the efficiency and the site effects of antiepileptic treatment. ${ }^{9-11}$ There appear to be few studies, however, on the prognosis of this syndrome in children.

The past pervasive tendency to consider the prognosis for partial seizures as much less favourable than that for generalised seizures has now been re-examined. Favourable outcomes of seizure symptomatology in childhood have been seen in at least two thirds of such patients. ${ }^{4}{ }^{12} 13$ There is favourable prognosis for social adjustment and intellectual development as well. Some authors found that one third or fewer patients had difficulties in social adjustment, whether or not they were associated with mental retardation. ${ }^{46}{ }^{14}$ In this study we have given special regard to the evolution of seizure symptomatology and to associated disturbances (that is, mental retardation, neurological abnormalities, behaviour and cognitive disorders) which compromise or hinder social adjustment. Furthermore we tried to establish which initial electroclinical characteristics and which course patterns have the greatest influence on seizure outcome, in order to define possible predictive factors for patients with this type of seizures.

\section{Patients and methods}

We studied 261 consecutive patients (136 males and 125 females) with partial epilepsy, who presented with seizures, on at least two separate occasions, that were not symptomatic of acute cerebral alterations of a febrile, toxic, or metabolic nature. The age of appearance of partial seizures ranged from 10 months to 13 years (average age 4.9 years). We excluded all patients with benign partial epilepsy ${ }^{15}$ or with partial epilepsy constantly associated with generalised seizures. The average duration of the follow up period was 7.4 years. All the patients were having antiepileptic treatment, and the blood concentrations were periodically reviewed; all the patients underwent at least semiannual clinical and electroencephalographic examinations (in waking and sleeping conditions). Eighty nine patients presented with partial seizures with elementary symptomatology; 109 with partial seizures with complex symptomatology; and 63 with partial seizures secondarily generalised. Aetiological prenatal, perinatal, or postnatal factors were present in 112 cases: 62 patients had birth injuries, 31 head traumas, and 19 infectious pathologies of the central nervous system with contemporary or subsequent onset of partial seizures.

We divided the whole sample into two main groups according to seizure outcome at the fifth follow up year. ${ }^{1}$ Improved cases included patients whose frequency of seizures was reduced by at least two thirds of the first year frequency and patients 
who presented stable remission of convulsive symptomatology for at least two years. ${ }^{2}$ Patients who were worse had an unchanged or a higher seizure frequency than at onset or had a seizure frequency not less than monthly.

We studied neurological and mental evolution in relation to the constant and stable presence of mental retardation of various levels (IQ <80), permanent neurological abnormalities (hemiplegia, tetraplegia, etc), behaviour disorders considerably affecting social adjustment (for example, childhood hyperkinesia, uncontrolled aggressiveness, etc), and cognitive disorders in absence of mental retardation (for example, speech and learning disabilities).

We observed the evolution of the electroencephalographic tracings in relation to the initial focal location; to possible regional, contralateral, or intrahemispheric migration; and to normalisation.

We investigated factors predictive of seizure outcome and of neurologic and mental evolution by correlating the trend of convulsive symptomatology at the end of the observation and the neurological and mental abnormalities, and behaviour and cognitive disorders, with the following initial and course characteristics: type of partial seizures, aetiology and/or positive family history of convulsions and/or epilepsy in first and/or second degree relatives, predating generalised seizures and febrile convulsions, age at partial seizure onset, initial seizure frequency, initial patterns on electroencephalography, frequency of seizures after 12 months treatment, duration of epilepsy (that is, the period between the first and the last partial seizure), and stable and persisting abnormalities in background activity on electroencephalography (such as diffuse stable delta activity).

Finally, we correlated seizure outcome with the patients' neurological, mental, and electroencephalographic results. We used the $\chi^{2}$ test for comparison of percentages, resorting to the Yates's correction factor when more than $20 \%$ of the observed numbers was equal to or lower than 5 . Differences in $p$ values equal to or lower than 0.05 were considered significant.

\section{Results}

(1) SEIZURE OUTCOME AT THE END OF THE FOLLOW UP PERIOD

At the end of the observation period, 153 patients had been free from seizures for at least two years; 61 had an improvement of initial seizure frequency; 36 had an unchanged seizure frequency; and 11 had a higher seizure frequency compared with that at onset.

\section{(2) EVOLUTION OF ELECTROENCEPHALOGRAPHIC TRACINGS AT THE END OF THE FOLLOW UP PERIOD (TABLE 1)}

In 109 patients the electroencephalographic tracings had become normal, 140 had focal specific abnormalities, and 12 had multifocal specific abnormalities.

During the follow up period transient multifocal activity was seen in 20 patients; this activity was then replaced by migrating focal activity. Of the whole sample, 83 patients presented subsequent migration of the electroencephalographic focus and 31 of them had normal tracings at the end of the follow up. Among the patients without subsequent migration of the initial focus (178) electroencephalography became normal in 77 . In most cases the initial locations of foci, subsequently normalised, were parietal, occipital, and frontal. The percentage becoming normal was very low, however, in subjects with focal initial location in the temporal area, both in the case of subsequently migrating foci $(12 / 45$, $27 \%$ ) and of foci stable in time $(29 / 76,38 \%)$. The rate that the tracings became normal did not differ in the two groups (constant foci and migrating foci) either in relation to the total number of cases or to single initial location of foci on electroencephalography.

Table 1 Location of initial foci, migration, and normalisation

\begin{tabular}{|c|c|c|c|c|c|c|}
\hline \multirow[t]{2}{*}{ Location } & \multicolumn{2}{|c|}{ Initial foci } & \multicolumn{2}{|c|}{ Migration } & \multicolumn{2}{|c|}{ No migration } \\
\hline & $\begin{array}{l}\text { Total } \\
\text { No }\end{array}$ & $\begin{array}{l}\text { Normalisation } \\
\text { No }(\%)\end{array}$ & $\begin{array}{l}\text { Total } \\
\text { No (\%) }\end{array}$ & $\begin{array}{l}\text { Normalisation } \\
\text { No }(\%)\end{array}$ & $\begin{array}{l}\text { Total } \\
\text { No }(\%)\end{array}$ & $\begin{array}{l}\text { Normalisation } \\
\text { No }(\%)\end{array}$ \\
\hline Frontal & 45 & $18(40)$ & $18(40)$ & $6(33)$ & $27(60)$ & $12(44)$ \\
\hline Parietal & 30 & $15(50)$ & $6(20)$ & $5(83)$ & $24(80)$ & $10(42)$ \\
\hline Occipital & 28 & $16(57)$ & $8(28)$ & $5(62)$ & $20(72)$ & $11(55)$ \\
\hline Temporal & 121 & $41(34)$ & 45 (37) & $12(27)$ & $76(63)$ & $29(38)$ \\
\hline Hemispheric & 31 & $18(58)$ & 6 (19) & $3(50)$ & $25(81)$ & $15(60)$ \\
\hline Multifocal & 6 & 1 (17) & - & - & $6(100)$ & $1(17)$ \\
\hline Total & 261 & 109 & 83 & 31 & 178 & 78 \\
\hline
\end{tabular}


(3) FACTORS INFLUENCING SEIZURE OUTCOME AND NEUROMENTAL EVOLUTION (PREDICTIVE FACTORS)

Data resulting from comparison of initial and course characteristics and final outcome of seizures are shown in table 2. No significance was achieved for febrile convulsions predating partial onset, seizure initial frequency, and the initial pattern on electroencephalography. With regard to neuromental evolution at the end of the follow up period 130 patients had at least one of the following disturbances: 44 patients had mental retardation, 49 neurological abnormalities, 45 behaviour disorders, and 43 cognitive disorders.

When we compared characteristics found initially and during the follow up period with mental retardation we found the following associations. (1) Type of seizure: partial seizures with elementary symptomatology, mental retardation was found in $17 / 89$ patients $(19 \%)$; partial seizures with complex symptomatology, 24/109 (22\%); and partial seizures secondarily generalised, $3 / 63(5 \%)(p<0 \cdot 025)$. (2) Aetiology: ascertained aetiology, $17 / 70$ patients (24\%); ascertained aetiology plus a positive family history, $13 / 42(31 \%)$; positive family history, 5/59 $(8 \%)$; and idiopathic forms, $9 / 90(10 \%)(p<0.005)$.
(3) Age at seizure onset: 0-4 years, 29/118 patients $(25 \%)$ and $4-12$ years, $15 / 143(11 \%)(p<0 \cdot 01)$. (4) Frequency of seizures after 12 months' treatment: high, $21 / 51$ patients $(41 \%)$; medium, $9 / 60(15 \%)$; and low, 14/150 (9\%) $(\mathrm{p}<0.0005)$. (5) Duration of epilepsy: two to four years, $16 / 147$ patients $(11 \%)$ and four to 13 years, $28 / 114(25 \%)(p<0.005)$. (6) Background activity on electroencephalography: present, $15 / 30$ patients $(50 \%)$ and absent, $29 / 231$ $(13 \%) \quad(p<0.0005)$. No differences were found for the initial frequency of seizures.

When we compared the characteristic found with neurological abnormalities we found the following associations. (1) Type of seizure: partial seizures with elementary symptomatology, neurological abnormalities were found in $24 / 89$ patients $(27 \%)$; partial seizures with complex symptomatology, 29/ $109(20 \%)$; and partial seizures secondarily generalised, $3 / 63(5 \%)(p<0 \cdot 005)$. (2) Aetiology: ascertained aetiology, $24 / 70$ patients $(34 \%)$; ascertained aetiology plus positive family history, $11 / 42(26 \%)$; positive family history, $6 / 59(10 \%)$; and idiopathic forms, $8 / 90(9 \%)(\mathrm{p}<0.005)$. (3) Age at seizure onset: $0-4$ years, $33 / 118$ patients $(28 \%)$ and $4-12$ years, $16 / 143(11 \%)(p<0 \cdot 001)$. (4) Frequency of

Table 2 Initial and course characteristics compared with seizure outcome*

\begin{tabular}{|c|c|c|c|c|}
\hline & $\begin{array}{l}\text { Total } \\
\text { No }\end{array}$ & $\begin{array}{l}\text { No (\%) who } \\
\text { improved } \\
(n=214)\end{array}$ & $\begin{array}{l}\text { No (\%) who } \\
\text { worsened } \\
\text { ( } n=47)\end{array}$ & p Value \\
\hline \multicolumn{5}{|l|}{ Type of seizures: } \\
\hline Partial elementary & 89 & $71(80)$ & $18(20)$ & \multirow{3}{*}{ NS } \\
\hline Partial complex & 109 & $85(78)$ & $24(22)$ & \\
\hline Partial secondarily generalised $\dagger$ & 63 & $58(92)$ & $5(8)$ & \\
\hline \multicolumn{5}{|l|}{ Aetiology: } \\
\hline Ascertained aetiology & 70 & $50(72)$ & $20(29)$ & \multirow{4}{*}{0.001} \\
\hline Ascertained aetiology + positive family history & 42 & $29(69)$ & $13(31)$ & \\
\hline Positive family history & 59 & $53(90)$ & $6(10)$ & \\
\hline Idiopathic forms & 90 & $82(91)$ & $8(9)$ & \\
\hline \multicolumn{5}{|l|}{ Generalised seizures predating partial onset } \\
\hline Present & 23 & $9(39)$ & $14(61)$ & \multirow{2}{*}{$0 \cdot 0005$} \\
\hline Absent & 238 & $205(86)$ & $33(14)$ & \\
\hline \multicolumn{5}{|l|}{ Age at onset: } \\
\hline $0-4$ years & 118 & $89(75)$ & $29(25)$ & \multirow{2}{*}{$0 \cdot 025$} \\
\hline 4-12 years & 143 & $125(87)$ & 18 (13) & \\
\hline \multicolumn{5}{|l|}{ Frequency of seizures after 12 months treatment $\ddagger$} \\
\hline High & 51 & $27(53)$ & $24(47)$ & \multirow{3}{*}{0.005} \\
\hline Medium & 60 & $49(81)$ & 11 (19) & \\
\hline Low & 150 & $138(92)$ & $12(8)$ & \\
\hline \multicolumn{5}{|l|}{ Duration of epilepsy: } \\
\hline $2-4$ years & 147 & $136(93)$ & $11(8)$ & \multirow{2}{*}{0.0005} \\
\hline $4-13$ years & 114 & $78(68)$ & $36(32)$ & \\
\hline \multicolumn{5}{|l|}{ Background activity on electroencephalography: } \\
\hline Present & 30 & $20(67)$ & 10 (33) & \multirow{2}{*}{0.025} \\
\hline Absent & 231 & $194(84)$ & 37 (16) & \\
\hline
\end{tabular}

*No significances were achieved for febrile convulsions predating partial onset, seizure initial frequency, and initial pattern on electroencephalography.

$\dagger$ Partial secondarily generalised $v$ partial elementary + partial complex, $p=0 \cdot 02$.

$\ddagger$ High=multidaily, daily, or weekly; medium =fortnightly, monthly, or quarterly; and low=six monthly or yearly. 
seizures after 12 months' treatment: high, 16/51 patients $(31 \%)$; medium, $9 / 60(15 \%)$; and low, $24 / 150(16 \%)(p<0.05)$. (5) Duration of epilepsy: two to four years, $21 / 147$ patients $(14 \%)$ and four to 13 years, 28/114 (25\%) (p<0.05). (6) Background activity on electroencephalography: present, $15 / 30$ patients $(50 \%)$ and absent, $34 / 231 \quad(15 \%)$ $(p<0 \cdot 005)$. No differences were found for the initial frequency of seizures.

When we compared the characteristics found with behaviour disorders we found the following associations. (1) Type of seizure: partial seizures with elementary symptomatology, behaviour disorders were found in 16/89 patients (18\%); partial seizures with complex symptomatology, 26/109 (24\%); and partial seizures secondarily generalised $3 / 63(5 \%)$ $(\mathrm{p}<0 \cdot 01)$. (2) Aetiology: ascertained aetiology, $17 / 70$ patients $(24 \%)$; ascertained aetiology plus a positive family history, $12 / 42(29 \%)$; positive family history, 6/59 (10\%); and idiopathic forms, 10/90 $(11 \%)(p<0.025)$. (3) Frequency of seizures after 12 months' treatment: high, $15 / 51$ patients $(29 \%)$; medium, 10/60 (17\%); and low, 20/150 (13\%) $(p<0.05)$. (4) Duration of epilepsy: two to four years, $13 / 147$ patients $(9 \%)$ and four to 13 years, $32 / 114(28 \%)(p<0.005)$. (5) Background activity on electroencephalography: present, $10 / 30$ patients $(33 \%)$ and absent, $35 / 231(15 \%)(p<0.025)$. No differences were found for the age at seizure onset and for the initial frequency of seizures.
When we compared the characteristics found with cognitive disorders, the only significant association was in the type of seizure, with partial seizures with elementary symptomatology $(18 / 89,20 \%)$ and partial seizures with complex symptomatology $(21 / 109$, $19 \%$ ) having a greater association than partial seizures secondarily generalised $(4 / 63,6 \%)$ $(\mathrm{p}<0 \cdot 05)$.

\section{(4) PARALLEL COMPARISON OF SEIZURE OUTCOME, NEUROLOGICAL AND MENTAL STATE, AND THE OUTCOME OF ELECTROENCEPHALOGRAPHIC TRACING}

Data are shown in table 3. No significant difference was found when we compared seizure outcome with neurological abnormalities, with foci migration shown on electroencephalography, and with the location of the initial foci shown on electroencephalography.

\section{Discussion}

In this series the outcome of patients with partial seizures was encouraging; remission of seizures for at least two years occurred in $59 \%$. On the total sample, $17 \%$ had mental retardation, $19 \%$ had neurological abnormalities, and $17 \%$ had behaviour disorders. These data match the observations reported by other authors. ${ }^{4} 12$

The results on electroencephalography and the clinical course tended to parallel each other,

Table 3 Comparison of seizure outcome, neurological and mental state, and outcome of electroencephalographic tracing*

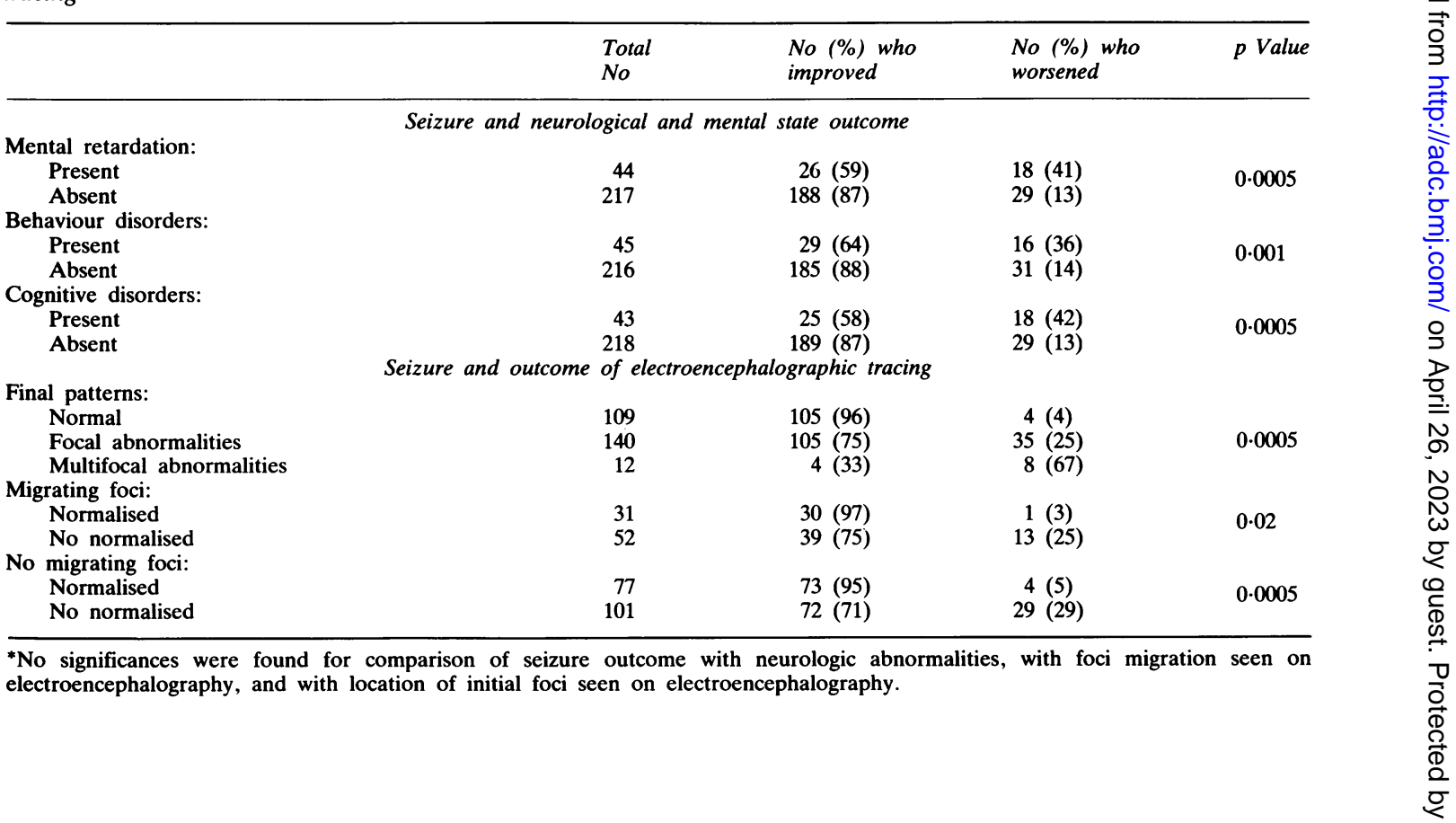


although the percentage of improvement in convulsive symptomatology exceeded the values of the normalisation of the electroencephalographic tracing. This finding is particularly stressed in other studies on partial seizures. ${ }^{412} 14$

The temporal area was the most frequent location of focus shown on electroencephalography in accordance with Scarpa. ${ }^{12}$ Patients with the focus in this area also presented the lowest value of normalisation on electroencephalography, such as that found in patients with multifocal tracings. Migration frequency of foci with initial temporal location did not differ from migration frequency of foci in the other locations, although the normalisation percentage was much lower. Patients with temporal foci that did not migrate had the lowest value of normalisation; however, a less favourable trend of convulsive symptomatology did not correlate with these values.

Focal migration was not, by itself, associated with tracing normalisation at the end of the follow up period, nor with improvement of convulsive symptomatology. Kitagawa has noted that temporal, multifocal, and migrating tracings tend to less favourable evolution. ${ }^{16}$

As for predictive factors of seizure outcome, we found that an ascertained aetiology led to unfavourable seizure outcome. The presence of both a positive family history and ascertained aetiology, however, led to poor outcome. It is possible that the addition of brain insults to the genetic disposition to convulsions is responsible for the increase and the persistence in time of a convulsive tendency. On the contrary, the absence of ascertained aetiology, and the presence of a positive family history were often associated with definitely favourable outcome, as other authors have found. ${ }^{12} 13$

Poor control of seizures, despite the introduction of antiepileptic treatment, was a very important factor in the subsequent evolution, whereas initial seizure frequency did not seem to influence prognosis. Other authors differ in their observation of this. $^{14}$

A normal initial tracing on electroencephalography had no prognostic values; in fact, in our sample, all the patients presenting normal initial tracings subsequently presented focal activity. The persistence of stable abnormalities in background activity on electroencephalography was an unfavourable influence on seizure outcome. This finding has already been reported in other studies on the prognosis of epilepsy and is thus confirmed as well in partial seizures of childhood. ${ }^{17}{ }^{18}$ Factors predictive of unfavourable seizure outcome were: early onset, presence of generalised seizures predating partial seizure onset, and long duration of epilepsy.
We found a more favourable seizure outcome for partial seizures secondarily generalised compared with partial seizures with elementary symptomatology and partial seizures with complex symptomatology. Some authors report a more favourable outcome for complex seizures, ${ }^{6}{ }^{18}$ and others agree with our results for partial generalised ones. ${ }^{14}{ }^{16} \mathrm{We}$ think that the presence of significant differences in the group with partial seizures secondarily generalised compared with the two other groups in our sample is due to the coexistence, in the groups with partial seizures with either elementary or complex symptomatology, of other types of partial seizures. In fact, it is well known that a stable association of various types of partial seizures is responsible, by itself, for a less favourable outcome. ${ }^{419}$

Predictive factors associated with unfavourable seizure trends were also associated with neurological abnormalities, mental retardation, and behaviour disorders. In many cases, patients with unfavourable seizure outcome were those presenting with an association of the above mentioned disorders. In our sample mental retardation and neurological abnormalities were less frequent than usual $(17 \%$ and $19 \%$ respectively), and they were evident before the onset of partial seizures. They were associated, but not causally related, with unfavourable seizure trends; this probably depends upon the amount of basic brain involvement, in accordance with the finding of Sofijanov. ${ }^{4}$ The low incidence of mental retardation and neurological abnormalities, and the lack of a causal relation between partial seizures and these pathologies, give evidence of the relative benignity of partial seizures in childhood.

There was no significant association between the presence of cognitive disorders and the characteristics found initially and throughout the follow up period. Speech and learning disabilities existed independently of characteristics linked with epileptic state. The percentage of cognitive disorders in our sample was similar to that in the general population. The outcome of partial seizures (even when benign epilepsies nosographically recognised are excluded) seems to be more favourable in childhood than among adults. We suggest that there exists a higher frequency of benign partial seizures among children, especially if they bear 'lesional' characteristics, than has previously been recognised. ${ }^{320}$

In conclusion, the main factors affecting a favourable prognosis are the following: (a) a family history positive for convulsions and the lack of ascertained aetiology; (b) absence of generalised seizures predating partial onset; (c) low seizure frequency after 12 months' treatment; (d) short duration of epilepsy; (e) absence of background activity abnor- 
malities on electroencephalography; and (f) absence of mental retardation, neurological abnormalities, or behaviour disorders. The following factors seem to have no determining influence on the prognosis: (a) presence of febrile convulsions predating partial onset-age at onset; (b) initial seizure frequency; (c) initial tracings on electroencephalography; and (d) presence of cognitive disorders.

\section{References}

1 Cavazzuti G B. Epilepsie de la seconde enfance (age scolaire). Congres de la Societe de Neurologie infantile. Marseille: Déffusion Generale de Librairie, 1977:219-27.

2 Viani F. Neuropsichiatria infantile. Epilessia. Disturbi relazionali. Utet, Torino Ed, 1981.

${ }^{3}$ Oller Daurella L, Oller F V L, Carol A, Russi A, Sanchez M E. Possibilidades de interrupcion del tratamiento antiepileptico. Bollettino della Lega Italiana contro l'Epilessia 1982;39:159-61.

4 Sofijanov N G. Clinical evolution and prognosis of childhood epilepsies. Epilepsia 1982;23:61-9.

5 Penry J K. Perspectives in complex partial seizures. In: Penry J K, Daly D D, eds. Advances in neurology. Vol II. New York: Raven Press, 1975:1-14.

${ }^{6}$ Lindsay J, Ounsted C, Richards P. Long term outcome in children with temporal lobe seizures. I: Social outcome and childhood factors. Dev Med Child Neurol 1979;21:285-98.

7 Russi-Tintoré A, Oller F V L, Oller Daurella L, Oller Sala J M. Analisis del fenomeno "cluster" y de los intervalos libres en crisis epilepticas partiales complejas. Bollettino della Lega Italiana contro l'Epilessia 1986;54/55:369-70.

${ }^{8}$ Shafer S, Hauser W A, Annegers J F, Kurland L T. Prognosis for control is not different for complex partial seizures than for all other seizure types in a community. Epilepsia 1986;27:615.

9 Tassinari C A, Roger J. Prognosis and therapy of partial seizures with barbiturates, hydantoins, and other drugs. In: Penry J K, Daly D D, eds. Advances in neurology Vol II. New York: Raven Press, 1975:201-16.

10 Roger J, Dravet C, Blanc-Baeri M J, Mesdjian E. Monothérapie par la carbamazepine dans le épilepsies partielles avec crisie à sémeiologie complexe. Bollettino della Lega Italiana contro l'Epilessia 1980;29/30:163-6.

1 Mitchell W G, Chavez J M. Carbamazepine versus phenobarbital for partial onset seizures in children. Epilepsia 1987;28:56-60.

12 Scarpa P, Carassini B. Partial epilepsy in childhood: clinical and EEG study of 261 cases. Epilepsia 1982;23:331-41.

13 Tsuji M, Morikawa T, Shigematsu H. Long term seizure prognosis of partial epilepsy in childhood. Epilepsia 1986;27: 617.

14 Okuma T, Kumshiro $H$. Natural history and prognosis of epilepsy: report of a multi-institutional study in Japan. Epilepsia 1981;22:35-53.

15 Gastaut H. Individualisation des épilepsies dites "bénignes" ou "founctionelles" aux differentes ages de la vie. Appreciation des variations correspondentes de la prédisposition épileptique a ces ages. Revue dé EEG et de Neurophysiologie Clinique 1982;II: 346-66.

16 Kitagawa T. A clinical and electroencephalographical follow-up study for more than 10 years in patients with epilepsy. Folia Psychiatr Neurol Jpn 1981;35:333-42.

${ }^{17}$ Laplane R, Salbreux R, Tubout A M, Deinard J M. 149 cas de convulsions dans le 18 premias mois de vie. Analyse statistique et correlations electrocliniques. Ann Pediatr (Paris) 1977;24:467-73.

${ }^{18}$ Fukushima Y. A longitudinal study on epilepsy: with special reference to recurrence of seizures after long seizure-free period. Folia Psychiatr Neurol Jpn 1981;35:343-8.

19 Yamada H, Yoshida H, Ninomiya H. A five year follow-up study of 66 epileptics. Folia Psychiatr Neurol Jpn 1981;35: 339-45.

20 Deonna T, Ziegler A L, Despland P A, van Melle G. Partial epilepsy in neurologically normal children: clinical syndromes and prognosis. Epilepsia 1986;27:241-7.

Correspondence to Professor G Porro, Instituto di Neuropsichiatr Infantile, Universita' 'La Sapienza' Roma, Via Dei Sabelli, 108, 00185 , Rome, Italy.

Accepted 31 May 1988 